\title{
Effects of fatigue on biomechanics of forehand smash in badminton
}

\author{
Shimin Zhang \\ Department of Physical Education, Xinjiang University of Finance and Economics, \\ Urumqi, Xinjiang, 830012, China \\ E-mail: zhangshimin@xjufe.edu.cn \\ Received 13 May 2020; received in revised form 23 June 2020; accepted 30 June 2020 \\ DOI https://doi.org/10.21595/jve.2020.21467
}

Check for updates

Copyright $(2020$ Shimin Zhang. This is an open access article distributed under the Creative Commons Attribution License, which permits unrestricted use, distribution, and reproduction in any medium, provided the original work is properly cited.

\begin{abstract}
Sports fatigue will cause deformation of actions. In this study, badminton was analyzed, and the influence which was brought by fatigue was studied from the perspective of biomechanics. The forehand smash of professional badminton athletes under normal and fatigue states was tested. The biomechanical indexes of athletes were obtained by infrared remote shooting test system and plantar pressure test system for comparison. The results showed that the forehand smash effect of athletes was significantly worse under fatigue state, the maximum gravity center height decreased to $1.22 \pm 0.14 \mathrm{~m}$, and the maximum gravity center speed decreased to $2.33 \pm 0.12 \mathrm{~m} / \mathrm{s}$, which showed significant decreases compared to the normal state $(P<0.05)$. Moreover the maximum velocity of the right upper arm, right forearm, elbow joint, wrist joint and knee joint decreased significantly $(P<0.5)$, the maximum angle of the right hip joint, knee joint and left ankle joint also decreased significantly. The pressure of the inner side of the forefoot and the pressure in the middle of the forefoot increased $(P<0.05)$. The biomechanical analysis of forehand smash under fatigue condition reveals the relationship between fatigue and movement, which provides some scientific bases for the reasonable control of sports load.
\end{abstract}

Keywords: badminton, forehand smash, fatigue, biomechanics.

\section{Introduction}

A large number of high-intensity sports are indispensable to all kinds of competitive sports, and fatigue is inevitable. The impact of fatigue on sports is also a matter of widespread concern for sports researchers. A previous study [1] has shown that fatigue can not only cause the decline of sports performance, but also is closely related to the increase of sports injury risk. Badminton is widely favored by people and has become one of the most popular sports in the world [2]. There are lots of high-intensity exercises in training or competition of badminton, a competitive sport; hence fatigue is inevitable. Understanding the impact of fatigue on the body is very important for athletes and coaches. Many studies have been carried out on the effects of fatigue on biomechanics. Schmitz et al. [3] found that fatigue could affect the stability of the knee joint and increased knee mobility and anterior cruciate ligament load, leading to anterior cruciate ligament injury. Fourchet et al. [4] took 11 young athletes as research subjects, found that leg stiffness decreased, vertical ground reaction force increased, and contact area and relative load of medial arch of foot increased significantly under fatigue condition $(P<0.01)$, indicating that fatigue could affect the protection of muscle and increase the risk of injury. Qu et al. [5] divided 32 male athletes into a fatigue-free group and a fatigue group and found that fatigue increased knee flexion during landing and knee rotation during cross-cutting, which possibly caused the risk of ACL injury. Jayalath et al. [6] analyzed jumping by two feet and jumping by one foot under fatigue. They found that fatigue affected the biomechanics of ankle joint, in details, the strength of ankle joint and ground reaction force were reduced, and the knee joint flexion was increased. Forehand smash is one of the most important and most used scoring methods in badminton [7,8]. It has characteristics of high speed, great strength and many routes. It is of great significance and value to study the biomechanical effects of forehand smash on badminton under fatigue condition. In 
this study, six professional badminton players were taken as the research subjects. The influence of fatigue on forehand smash was studied from the biomechanical perspective. The biomechanical index data of athletes under different conditions were obtained and compared through experiments, in order to understand the influence of fatigue on the completion of athletes' movements and provide some reference for reasonable adjustment of fatigue state.

\section{Fatigue}

A large number of high-intensity sports will inevitably lead to sports fatigue, which will not only affect sports performance, but also increase the risk of sports injury [10]. Fatigue refers to the inability of the body to maintain a certain level of function or a predetermined intensity of exercise [11]. The main mechanism of fatigue is the central fatigue mechanism (energy consumption of central nervous cells leads to a decline in body vitality) and the peripheral fatigue mechanism (changes in peripheral intracellular and extracellular fluids lead to a decline in muscle working ability). It can be divided into acute fatigue and chronic fatigue according to time and also can be divided into local fatigue and global fatigue according to location. When the badminton athletes compete or train to some extent, the organ functions of athletes will temporarily decline, leading to exercise induced fatigue. Exercise induced fatigue is an acute, global fatigue.

The criteria of fatigue include the attenuation rate of vertical jump height and the change of heart rate. In this study, scale of rating of perceived exertion (RPE) [12] which originates from Brog, a Swedish psychologist, was used as the criterion of fatigue judgment. The scale is formulated according to principles of psychology and is used for judging the self perception and loading of subjects.

The specific content is shown in Table 1.

Table 1. RPE scale

\begin{tabular}{|c|c|c|}
\hline RPE grade & Perceived exertion & Corresponding heart rate \\
\hline 6 & Quiet & \\
\hline 7 & Extremely relaxed & 70 \\
\hline 8 & & 90 \\
\hline 9 & Very relaxed & 110 \\
\hline 10 & & \\
\hline 11 & Relaxed & 130 \\
\hline 12 & & 150 \\
\hline 13 & Slightly strenuous & \\
\hline 14 & & 170 \\
\hline 15 & Strenuous & \\
\hline 16 & & \\
\hline 17 & Very strenuous & \\
\hline 18 & & \\
\hline 19 & Extremely strenuous & \\
\hline 20 & & \\
\hline
\end{tabular}

\section{Research subjects}

The research subjects of this study were six professional badminton players from the Men's Badminton Team of Central South University of Finance, Economics and Law, Hubei, China. They held rackets with the right hand and were healthy and skilled in forehand smash techniques. They had no intense exercise, took the same diet, did not intake ethyl alcohol or caffeine, and had good mental state in 24 hours before the experiment. They fully warmed up for 30 minutes before the experiment. The basic information of the subjects is shown in Table 2. 
Table 2. The basic information of the research subjects

\begin{tabular}{|c|c|c|c|c|}
\hline No. & Age & Height $/ \mathrm{m}$ & Weight / kg & Training time / year \\
\hline 1 & 22 & 178 & 78 & 3 \\
\hline 2 & 24 & 179 & 80 & 4 \\
\hline 3 & 23 & 176 & 80 & 3 \\
\hline 4 & 25 & 181 & 81 & 3 \\
\hline 5 & 26 & 178 & 81 & 4 \\
\hline 6 & 24 & 180 & 80 & 3 \\
\hline
\end{tabular}

\section{Research methods}

\subsection{Experimental equipment}

QUALISYS-MCU500 Infrared long shot testing system (Qualisys, Sweden) which has six lens and 240 frames per second was used, and Novel Pedar System (Novel Pedar, Germany) with a sampling frequency of $100 \mathrm{~Hz}$ was used for collecting the changes of plantar pressure in the period of landing.

\subsection{Experimental flow}

(1) The height and angle of the lens were adjusted to ensure that the shooting range could completely cover the athletes' range of motion.

(2) The test space was calibrated to ensure that the accuracy error did not exceed $3 \%$.

(3) After warming up, the subjects wore special shoes for experiment. The insoles had no crease, and the size of the insoles matched the shoes perfectly. The receiver of the sole pressure testing system was placed at the waist of the subjects. Then the researchers pasted the reflective marking points on the shoulder, right elbow, right wrist, right hand, hip joint, knee joint, ankle joint and the top of the racket.

(4) Every athlete tried his best to do in-situ forehand smash for three times. The main points of action included facing the ball on the right side of the body, hitting the ball with the racket face on one side of the palm, full coordination of joints during hitting, exerting force through the details of the wrist and fingers at the moment of hitting, hitting the ball with the upper part of the net, hitting the ball forward, following inertia after hitting, swinging the right hand towards the lower left direction, keeping center of gravity of lower limbs stable and returning to the central position for next hitting.

(5) On Monark 839E power bicycle, the incremental load exercise was carried out. The initial load was $90 \mathrm{~W}$, and the incremental speed was $30 \mathrm{~W}$ in every two minutes. The fatigue was judged by RPE scale. When RPE was greater than or equal to 19, the exercise was stopped. Each athlete immediately completed three full-strength forehand smash movements.

\subsection{Data processing}

The three-dimensional coordinates of the reflective marking points were obtained by the QTrc software of QUALISYS test system. The data were smoothed by the numerical filtering method. Moreover, data were obtained by the plantar pressure test system. The plantar pressure insoles were divided into nine parts as shown in Fig. 1 and Table 3. The obtained data were analyzed using EXCEL software and SPSS13.0 software. Average values of three times of actions were taken as the data of different indexes. Graphs and tables were drawn, and abstract data were statistically processed. 


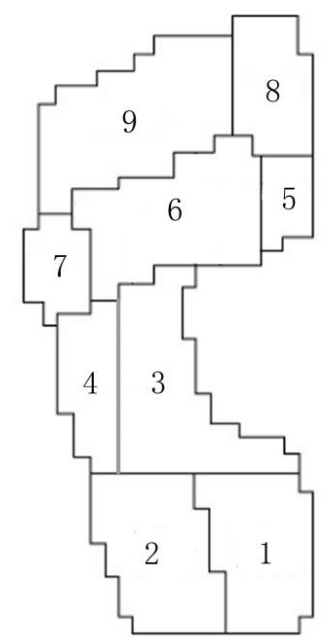

Fig. 1. Division of plantar pressure insole

Table 3. Division of plantar pressure insole

\begin{tabular}{|c|c|}
\hline No. & Site \\
\hline 1 & Inner side of rearfoot \\
\hline 2 & Outer side of rearfoot \\
\hline 3 & Inner side of middle foot \\
\hline 4 & Outer side of midfoot \\
\hline 5 & Inner side of midfoot \\
\hline 6 & Middle of forefoot \\
\hline 7 & Outer side of forefoot \\
\hline 8 & Big toe \\
\hline 9 & Other toes \\
\hline
\end{tabular}

\section{Research results}

\subsection{Changes of forehand smash effect}

It was seen from Table 4 that the forehand smash effect of the athletes had significant changes before and after fatigue. The racket speed was $38.64 \pm 3.16 \mathrm{~m} / \mathrm{s}$ during hitting, and the speed of ball over the net was $18.69 \pm 3.45 \mathrm{~m} / \mathrm{s}$ in the fatigue state, which were significantly decreased compared to the normal state $(P<0.05)$; the landing time of ball was $0.51 \mathrm{~s}$. The landing time of ball was $0.18 \mathrm{~s}$ in the normal state. It indicated that the forehand smash effect of the athletes became much worse in the fatigue state, leading to small ball speed, large air resistance, long path and small threaten.

Table 4. Comparison of forehand smash effect

\begin{tabular}{|c|c|c|}
\hline Indicator & Normal & Fatigue \\
\hline Racket speed during hitting $(\mathrm{m} / \mathrm{s})$ & $41.27 \pm 3.92$ & $38.64 \pm 3.16^{*}$ \\
\hline Speed of ball over the net $(\mathrm{m} / \mathrm{s})$ & $41.39 \pm 2.67$ & $18.69 \pm 3.45^{*}$ \\
\hline Landing time of ball (s) & 0.18 & $0.51^{*}$ \\
\hline
\end{tabular}

\subsection{Changes of gravity center indicators}

Table 5 shows the changes of the maximum height and speed of gravity center when the athletes were in the normal and fatigue states. Figs. 2 and 3 show the curves of the height and speed of gravity center with time when an athlete completes forehand smash. It was seen from 
Table 5 that the maximum height and speed of the center of gravity of the athletes in the fatigue state were $1.22 \pm 0.14 \mathrm{~m}$ and $2.33 \pm 0.12 \mathrm{~m} / \mathrm{s}$ respectively, which were significantly lower than those in the normal state $(P<0.05)$. It was seen from Fig. 2 and 3 that the height of gravity center in the fatigue state was lower and the speed of the center of gravity was smaller in the whole movement process in the fatigue state, indicating that fatigue could affect the performance of the athletes in doing forehand smash action.

Table 5. Change of gravity center indicators

\begin{tabular}{|c|c|c|}
\hline Indicator & Normal & Fatigue \\
\hline The maximum height of gravity center $(\mathrm{m})$ & $1.37 \pm 0.21$ & $1.22 \pm 0.14^{*}$ \\
\hline The maximum speed of gravity center $(\mathrm{m} / \mathrm{s})$ & $2.78 \pm 0.52$ & $2.33 \pm 0.12^{*}$ \\
\hline$*: P<0.05$ compared to the normal state \\
\hline
\end{tabular}

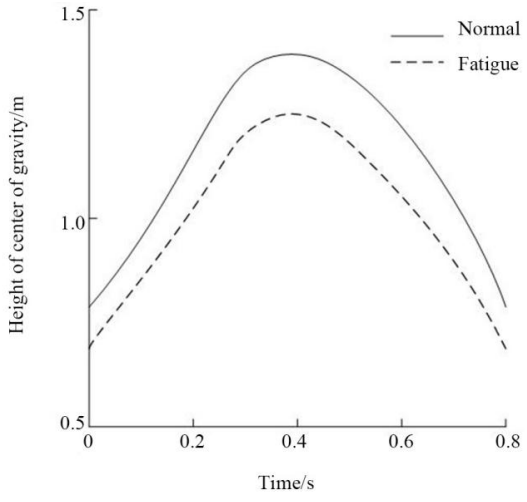

Fig. 2. The curve of changes of height of gravity center

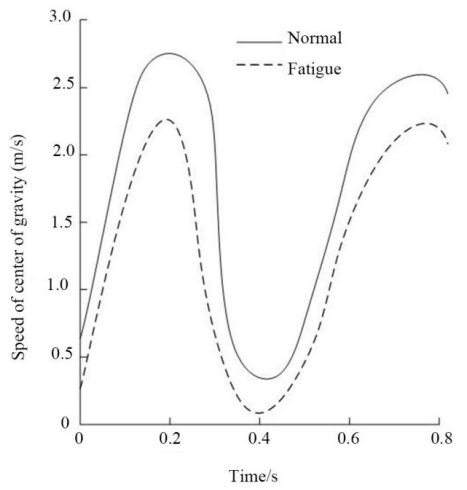

Fig. 3. The curve of changes of speed of gravity center

\subsection{Changes of biomechanics of upper limbs}

It was seen from Fig. 4 that different indicators of the upper limbs showed different degrees of decline in the fatigue state. The maximum speed of the right upper arm and forearm declined to $4.23 \pm 0.15 \mathrm{~m} / \mathrm{s}$ and $5.36 \pm 0.57 \mathrm{~m} / \mathrm{s}$ respectively, which was significant compared to those in the normal state $(P<0.05)$. The decline of the maximum speed of the shoulder joint was insignificant $(P>0.05)$. The maximum speed of the elbow joint and wrist joint declined to $7.36 \pm 0.74 \mathrm{~m} / \mathrm{s}$ and $10.29 \pm 1.27 \mathrm{~m} / \mathrm{s}$ respectively, which was significant $(P<0.05)$. Different indicators of the lower limbs had a large influence on the performance of forehand smash. In the fatigue state, the speed of arms decreased, and the speed of different joints also decreased, leading to the weakened ability of the wrist and fingers in controlling the angle of battledore, which could affect the flight speed and track of the ball.

\subsection{Changes of biomechanics of the lower limbs}

Table 6 shows the comparison of the biomechanical indicators of the lower limbs in the normal and fatigue state. It was found that the maximum angle of the right hip joint significantly decreased $(P<0.05), 28.21 \pm 3.11^{\circ}$. The maximum angle and speed of the knee joint significantly decreased $(P<0.05)$, and the maximum angle of the left ankle joint also significantly decreased $(P<0.05)$, $68.24 \pm 3.91^{\circ}$. The change of the hip joint was related to the flexion, extension and torsion of the body. In the fatigue state, the angle of the hip joint became smaller and the extension of the hip joint was inadequate, which led to the decrease of the range of motion of other links of the lower limbs. The change of the knee joint and ankle joint made the transmission speed of the lower limbs worse, the strength of the lower limbs smaller, the take-off height decrease and the hitting point 
lower; hence the performance was worse in the fatigue state.

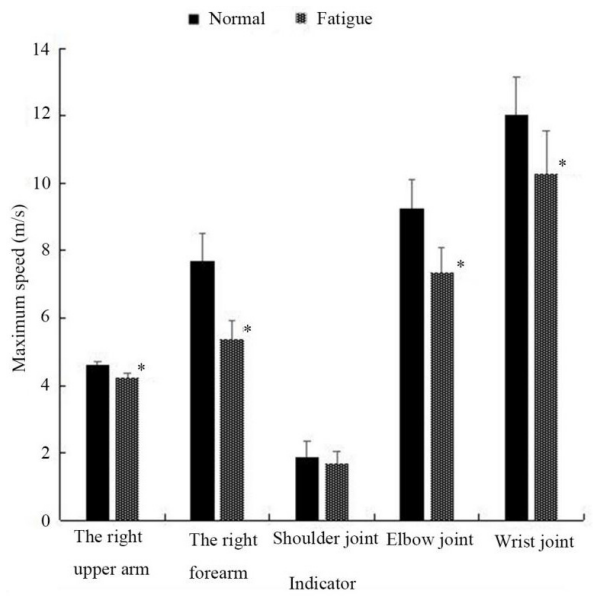

Fig. 4. Changes of indicators of upper limbs, $*: P<0.05$ compared to the normal state

Table 6. Changes of different indicators of the lower limbs

\begin{tabular}{|c|c|c|c|}
\hline \multicolumn{2}{|c|}{ Indicator } & Normal & Fatigue \\
\hline \multirow{2}{*}{ The maximum angle of the hip joint $\left({ }^{\circ}\right)$} & The left hip & $25.86 \pm 3.12$ & $24.27 \pm 3.27$ \\
\cline { 2 - 4 } & The right hip & $31.92 \pm 2.46$ & $28.21 \pm 3.11^{*}$ \\
\hline \multirow{2}{*}{ The maximum speed of the hip joint $(\mathrm{m} / \mathrm{s})$} & The left hip & $2.78 \pm 0.39$ & $2.73 \pm 0.29$ \\
\cline { 2 - 4 } & The right hip & $2.81 \pm 0.41$ & $2.67 \pm 0.28$ \\
\hline \multirow{2}{*}{ The maximum angle of the knee joint $\left({ }^{\circ}\right)$} & The left knee & $175.29 \pm 5.34$ & $164.31 \pm 4.37^{*}$ \\
\cline { 2 - 4 } & The right knee & $170.64 \pm 6.27$ & $156.74 \pm 5.28^{*}$ \\
\hline \multirow{2}{*}{ The maximum speed of the knee joint $(\mathrm{m} / \mathrm{s})$} & The left knee & $3.74 \pm 0.57$ & $3.12 \pm 0.48^{*}$ \\
\cline { 2 - 4 } & The right knee & $3.84 \pm 0.36$ & $3.07 \pm 0.29^{*}$ \\
\hline \multirow{2}{*}{ The maximum angle of the ankle joint $\left({ }^{\circ}\right)$} & The left ankle & $132.46 \pm 4.58$ & $68.24 \pm 3.91^{*}$ \\
\cline { 2 - 4 } & The right ankle & $131.94 \pm 4.66$ & $126.48 \pm 4.34$ \\
\hline \multirow{2}{*}{ The maximum speed of the ankle joint $(\mathrm{m} / \mathrm{s})$} & The left ankle & $5.46 \pm 1.12$ & $5.02 \pm 0.97$ \\
\cline { 2 - 4 } & The right ankle & $5.56 \pm 0.94$ & $5.11 \pm 1.08$ \\
\hline$*: P<0.05$ compared to the normal state & & \\
\hline
\end{tabular}

\subsection{Changes of plantar pressure}

It was seen from Fig. 5 that the maximum plantar pressure increased greatly or slightly in the fatigue state, which indicated that fatigue made the lower limbs fail to disperse the stress in time, and the stress was concentrated on the pelma, which increased the risk of ankle and knee joint injury. The pressure of area 5 (inner side of forefoot) increased from $173 \mathrm{MPa}$ to $242 \mathrm{MPa}$, and that of area 6 (middle of forefoot) increased from $181 \mathrm{MPa}$ to $236 \mathrm{MPa}$; differences were statistically significant $(P<0.05)$. In the other areas, the pressure in the fatigue state also increased, but the difference was not significant. The maximum pressure of the inner side of forefoot and the middle of forefoot in the fatigue state was significantly higher than that in the normal state. The increased pressure of the inner side of forefoot and middle of forefoot might increase the risk of injury of metatarsal bone in the inner side of forefoot and affect knee joint injury.

\section{Discussion and conclusion}

Sports induced fatigue is inevitable [13], especially for professional athletes. Badminton is a skilled sport. Badminton professional athletes need a strong technical level and a comprehensive 
physical quality, which requires the accurate coordination of the upper limns, body and lower limbs [14]. Competition time of badminton may be half an hour or more than one hour, the interval time is short and frequent, and the intensity and amount of motion are large, leading to large consumption of physical energy.

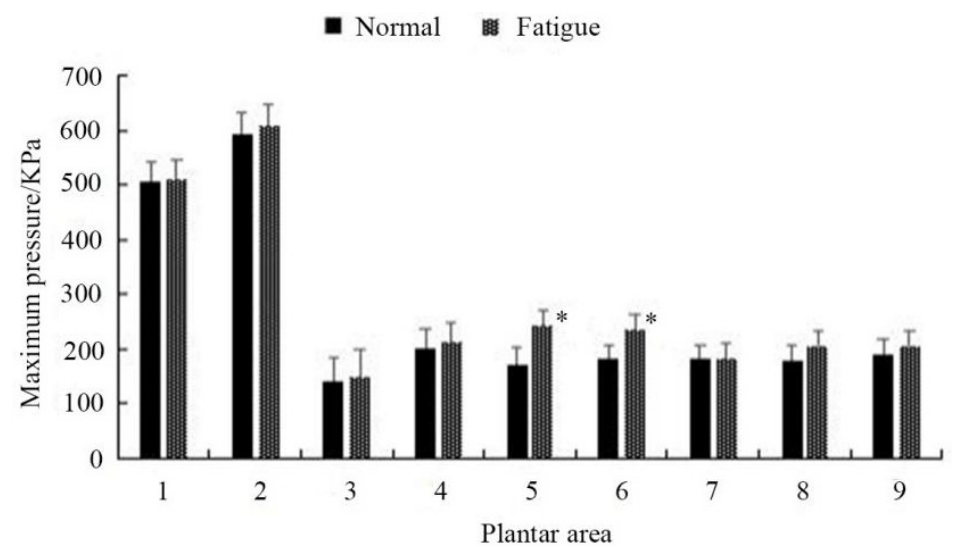

Fig. 5. The influence of fatigue on plantar pressure, $*: P<0.05$ compared to the normal state

By comparing the biomechanical indicators of the athletes in normal and fatigue states, it was found that the performance of the athletes declined significantly in the state of fatigue. Forehand smash is a process in which the upper arm drives the forearm. In the fatigue state, the maximum speed of different links of the upper arm reduces, the upper arm and forearm are not powerful enough, so that the maximum speed is not reached at the moment before hitting the ball, resulting in poor effect. In the process of movement, the lower limb mainly stores the elastic potential energy through the joint flexion to obtain the flight time and bounce height, so as to form a reasonable hitting posture and the highest hitting point. According to the results, it was found that the maximum angle and speed of the lower limb joint of the athletes decreased under the fatigue state, so it was difficult to obtain a high hitting point, which was not conducive to the full completion of the action. In addition, it was found from the analysis of plantar pressure that the plantar pressure of athletes increased significantly with the deepening of fatigue, which was because that the coordination ability of athletes decreased under the influence of fatigue and different links of lower limbs could not disperse fatigue in time. The medial part of forefoot and the middle part of forefoot bore the maximum pressure before and after fatigue, which was to provide sufficient support and buffer for feet; in the fatigue state, in order to ensure the stability of the foot, the pressure on the two regions bore more supporting role and greater risk of damage.

This study has verified that fatigue would have a negative impact on badminton athletes when doing the action of forehand smash. Therefore, in the process of training and competition, athletes should learn to control the consumption of physical strength, adjust fatigue in time, pay more attention to the standardization of movement after fatigue, and reduce the possibility of sports injury. Moreover, the research results also provide some theoretical support for coaches to make reasonable sports plans and relieve the fatigue of athletes in time, which can be popularized in practice to promote the long-term development of athletes.

\section{References}

[1] Liederbach M., Kremenic I. J., Orishimo K. F., et al. Comparison of landing biomechanics between male and female dancers and athletes, part 2: influence of fatigue and implications for anterior cruciate ligament injury. American Journal of Sports Medicine, Vol. 42, Issue 5, 2014, p. 1089-1095.

[2] Phomsoupha M., Laffaye G. The science of badminton: game characteristics, anthropometry, physiology, visual fitness and biomechanics. Sports Medicine, Vol. 45, Issue 4, 2015, p. 473-495. 
[3] Schmitz R. J., Kim H., Shultz S. J. Neuromuscular fatigue and tibiofemoral joint biomechanics when transitioning from non-weight bearing to weight bearing. Journal of Athletic Training, Vol. 50, Issue 1, 2015, p. 23.

[4] Fourchet F., Taiar R., Millet P. G. Influence of fatigue on running biomechanics in adolescent athletes. Annals of Physical and Rehabilitation Medicine, Vol. 56, Issue 1, 2013, p. 212.

[5] Qu X., Jiang J., Hu X. Effects of sub-sensory noise and fatigue on knee landing and cross-over cutting biomechanics in male athletes. Journal of Applied Biomechanics, Vol. 34, Issue 3, 2018, p. 205-210.

[6] Jayalath J. L. R., Denoronha M., Weerakkody N., et al. Effects of fatigue on ankle biomechanics during jumps: A systematic review. Journal of Electromyography and Kinesiology Official Journal of the International Society of Electrophysiological Kinesiology, Vol. 42, 2018, p. 81.

[7] Li S., Zhang Z., Wan B., et al. The relevance of body positioning and its training effect on badminton smash. Journal of Sports Sciences, Vol. 35, Issue 4, 2016, p. 310-316.

[8] Wei W., Ying S. Research on technical and tactical characteristics of LIN Dan in 2016 all England badminton open. Journal of Hubei Normal University, Vol. 37, Issue 3, 2017, p. 22-28.

[9] Gang L., Zhongqiu J., Xulong L. The biomechanical analysis on forehand smash of Male badminton players. Contemporary Sports Technology, Vol. 4, Issue 26, 2014, p. 7-10.

[10] Xu X., Ding Y., Yang Y., et al. $\beta$-glucan salecan improves exercise performance and displays anti-fatigue effects through regulating energy metabolism and oxidative stress in mice. Nutrients, Vol. 10, Issue 7, 2018, p. 858.

[11] Alaska A. L., Meng Q. C., Liu Z. F. Uighur medicine humoral quality of dialectical analysis of exercise-induced fatigue. Chinese Journal of Ethnomedicine and Ethnopharmacy, Vol. 22, Issue 14, 2013, p. 26-27.

[12] Gupta B. Ergonomic soft mouse and armrest mouse pad. Ubiquity, 2004, https://doi.org/10.1145/1029383.1029385.

[13] Ma J., Chen H., Liu X., et al. Exercise-induced fatigue impairs bidirectional corticostriatal synaptic plasticity. Frontiers in Cellular Neuroscience, Vol. 12, 2018, p. 14.

[14] Huang M. T., Lee H. H., Lin C. F., et al. How does knee pain affect trunk and knee motion during badminton forehand lunges? Journal of Sports Sciences, Vol. 32, Issue 7, 2014, p. 690-700.

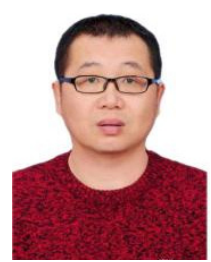

Shimin Zhang has gained the master's degree. He is a lecturer in Xinjiang University of Finance and Economics, China. He is interested in physical education teaching and sports humanistic sociology. 